\title{
AN ADAPTIVE FILTER BASED ON LOCAL SLOPE BY ADJUSTING ELLIPTIC FILTER KERNEL FOR ICESAT-2 PHOTON COUNTING LASER ALTIMETRIC DATA

\author{
Huan Xie ${ }^{1,}$, Dan Ye ${ }^{1}$, Gang Hai ${ }^{1}$, Xiaohua Tong ${ }^{1}$
} \\ ${ }^{1}$ College of Surveying and Geo-informatics, Tongji University, 1239 Siping Road, Shanghai 200092, \\ P. R. China
}

*Email: huanxie@tongji.edu.cn

\begin{abstract}
The Ice, Cloud and Land Elevation Satellite-2 (ICESat-2) was launched on September 15th, 2018, which continues to perform measurement tasks as the successor to ICESat. Unlike full waveform technology of ICESat, ICESat-2 employs micropulse photon counting technology, which provides higher accuracy, but produces plenty of noise. This paper proposes an adaptive filter based on local slope by adjusting elliptic filter kernel. The general approach is 1) data preprocessing, 2) Gaussian density calculation, 3) OTSU adaptive threshold calculation. This method is seen to be robust in identifying signal points from high background noise points and suitable for low density data caused by slope.
\end{abstract}

Index Terms - photon counting, noise filtering, Gaussian density, ICESat-2

\section{INTRODUCTION}

Follow-up mission to ICESat, the main science objectives of ICESat-2 are measuring ice sheet changes, sea ice thickness and vegetation biomass $^{[1]}$. ICESat-2 is equipped with the Advanced Topographic Laser Altimeter System (ATLAS), which collects elevation data using photon counting technology. The photon counting LiDAR has high level of sensitivity and high repetition frequency, but needs low pulse energy, which makes it possible to run at a higher altitude and provide larger coverage ${ }^{[2]}$. ICESat- 2 is also the first time to equip a photon counting LiDAR on a satellite for Earth observation.

The ATLAS instrument adopts a laser pulse in the green $(532 \mathrm{~nm})$, which is spilt into 6 beams organized in 3 pairs. The distance is $3.3 \mathrm{~km}$ between each pair and $90 \mathrm{~m}$ inside each pair. Each pair consists of a strong and a weak beam, and the strong is 4 times as the weak. The sensor uses a 10 $\mathrm{kHz}$ repetition rate $(\sim 0.7 \mathrm{~m}$ on the ground $)$ at the altitude of $\sim 500 \mathrm{~m}$ and produce $\sim 17 \mathrm{~m}$ overlapping footprint ${ }^{[3]}$. The data is discrete point clouds, which mainly come from target reflection, atmospheric scattering, solar background noise and the instrument dark count ${ }^{[4]}$. An efficient filter method for photon counting LiDAR data is extremely important to obtain ground information.

In 2014, a density-based clustering method is proposed $^{[5]}$. This method changes the shape of search area from a circle to an ellipse and calculated local area density. Subsequently the signal points were extracted according to the threshold of density. It could effectively extract ground and canopy, but did not consider the impact of surface slope. For inclined surface, the direction of ellipse consistent with the slope can get better clustering results. This paper changes the direction of ellipse according to surface slope. Since nearground noise points are difficult to distinguish from ground points, we give a greater weight in the direction of the track when calculating the density, and adopt the Gaussian density. Because the size of the laser footprint varies with the slope, the size of the elliptical nucleus also varies depending on the slope. Finally, the OTSU adaptive threshold method $^{[6]}$ is used to obtain a threshold of density for filter.

\section{METHODOLOGY}

In this paper, an improved adaptive filter method is proposed, as shown in Figure 1.

\subsection{Preprocessing}

Due to the edge effect, the density of points in the bounding area are smaller, the edge points are supplemented by mirror image method ${ }^{[7]}$.

\subsection{The elliptical Gaussian density calculation}

Since the signal points are tightly distributed, like a line, and the density is greater than the noise, the 
filter kernel has changed to an ellipse ${ }^{[5]}$. The size of semi-major axis $a$ and semi-minor axis $b$ of the ellipse at $p$ point were determined by the topographical features and the ground reflectivity.

$$
\begin{gathered}
\operatorname{dist}(p, q)=\left[\frac{\Delta X_{\theta}{ }^{2}}{a^{2}}+\frac{\Delta h_{\theta}{ }^{2}}{b^{2}}\right]^{\frac{1}{2}} \\
\left\{\begin{array}{l}
\Delta X_{\theta}=\cos \theta\left(X_{p}-X_{q}\right)+\sin \theta\left(h_{p}-h_{q}\right) \\
\Delta h_{\theta}=\cos \theta\left(h_{p}-h_{q}\right)-\sin \theta\left(X_{p}-X_{q}\right)
\end{array}\right.
\end{gathered}
$$

Where: $\theta$ is the slope of the center $\mathrm{p}, X_{p}$ and $X_{q}$ are the distances along the track, and $h_{p}$ and $h_{q}$ are the elevations. If $\operatorname{dist}(p, q)$ is less than 1 , the point is inside the ellipse, else outside the ellipse.

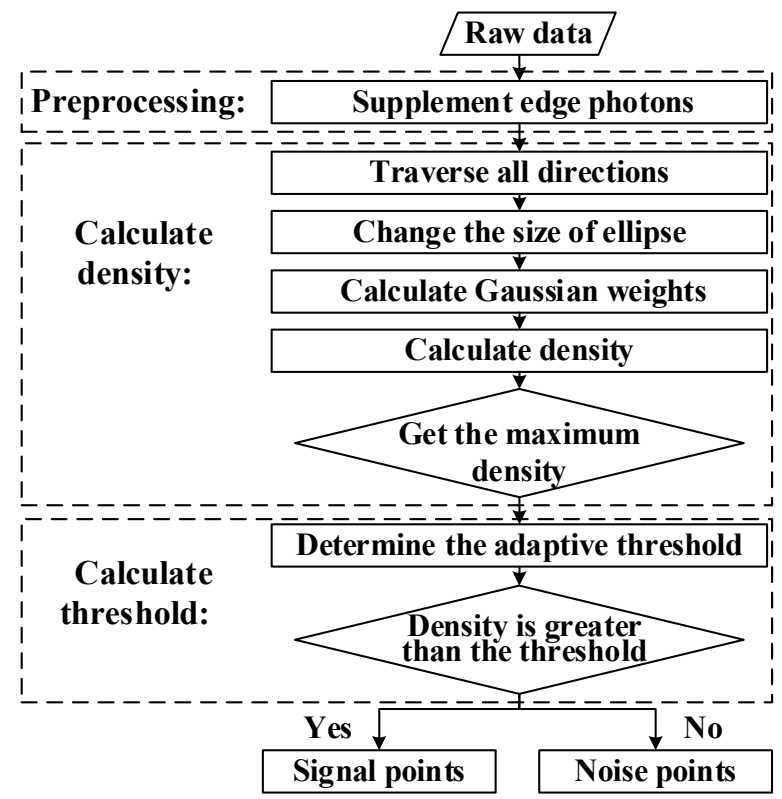

Fig 1. The flow chart of the elliptical Gaussian density adaptive filter method

Since the laser light irradiate on the slope and the distribution of laser energy varies, the size of the ellipse also varies accordingly.

Centering on each point, the points within the ellipse are given Gaussian weights ${ }^{[8]}$. The sum of the weights of all points in the ellipse is the density of the center point. There is the highest density along the tangential direction of the ground for ground points. Traversing all directions, the direction $\theta_{i}$ in which has the maximum density value is considered to be the slope direction, and the direction of filter kernel is set to $\theta_{i}$.

\subsection{OTSU adaptive threshold calculation}

The OTSU method is an algorithm for determining the image binarization threshold. In principle, the method is called the maximum inter-class variance method. Because the image binarization is divided according to the threshold obtained by the OTSU method, the variance between the foreground and the background image is the largest. In this paper, we use the OTSU method to obtain the density adaptive threshold and extract the signal points.

\section{EXPERIMENT AND RESULTS}

Because the ICESat-2 data has not yet been released, this paper use its onboard simulation data MABEL (the Multiple Altimeter Beam Experimental Lidar) instead. In order to verify that the algorithm is suitable for terrain with slope, we chose the MABEL data with a steep terrain gathered on August 2, 2014.

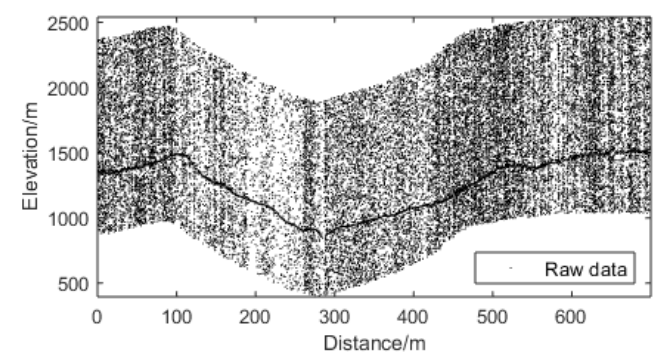

Fig 2. Profile of raw data, the x-coordinate is the distance along the track, and the y-coordinate is the elevation

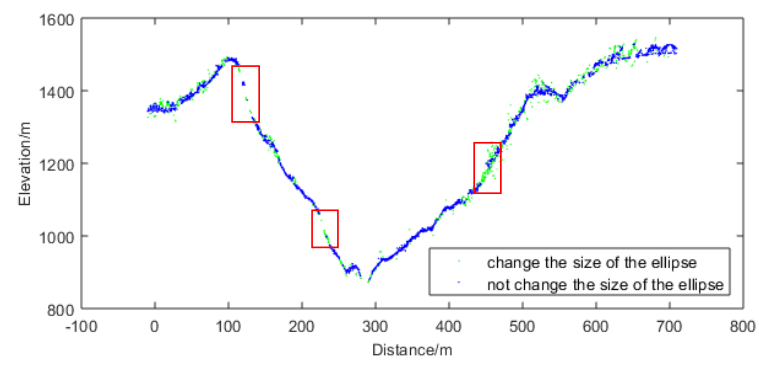

Fig 3. Results of signal points extraction (the green dots are the result of the ellipse size according to the slope, and the blue don't change)

The results of signal points extraction are shown in Figure 3. Parameters used in this paper are: $a=$ $10, b=2$, Gaussian weight variance $\sigma=2$. Here the blue dots represent the result without changing the size of the cluster ellipse, and the green dots represents the filter result of the ellipse size according to the gradient. The results within the red 
rectangle show that the method is more effective for extracting signal points on the slope surface.

\section{CONCLUSION}

In this paper, the existing elliptical cluster filter method is improved. In terms of density calculation, the near-ground noise points and the ground points are considered very close. This paper uses the Gaussian density to give a greater weight in the direction of the track. Secondly, since the footprint and energy of the laser vary in the slope, the density becomes smaller, and we change the ellipse size according to the slope. The OTSU method is used to determine an adaptive threshold. The results show that the filter method in this paper have some improvement in the slope.

\section{ACKNOWLEDGEMENTS}

This work was supported by the National Natural Science Foundation of China under Grant No. 41822106 and 41571407, National High Resolution Ground Observation System of China (GF-7) under Grant No. 11-Y20A12-9001-17/18, Science and Technology Innovation Action Plan Program of Shanghai under Grant No. 18511102100, Dawn Scholar of Shanghai under Grant No. 18SG22, and the Fundamental Research Funds for the Central Universities of China.

\section{REFERENCES}

[1] W. Abdalati et al., "The ICESat-2 Laser Altimetry Mission," Proceedings of the IEEE, 98(5): 735-751 (2010)

[2] J. J. Degnan, "Photon-counting multikilohertz microlaser altimeters for airborne and spaceborne topographic measurements," Journal of Geodynamics, 34(3): 503-549 (2002)

[3] L. A. Magruder et al., "Performance Analysis of Airborne Photon- Counting Lidar Data in Preparation for the ICESat-2 Mission," IEEE Transactions on Geoscience and Remote Sensing, 56(5): 2911-2918 (2018)

[4] F. Xie et al., "An adaptive directional filter for photon counting Lidar point cloud data" (in Chinese), Journal of Infrared and Millimeter Waves,1: 107-113 (2017)

[5] J. Zhang et al., "A clustering approach for detection of ground in micropulse photon-counting LiDAR altimeter data," in Geoscience and Remote Sensing Symposium, 177-180 (2014)

[6] N. Ohtsu, "A Threshold Selection Method from Gray-Level Histograms," IEEE Transactions on Systems Man \& Cybernetics, 9(1): 62-66 (1979)

[7] S. Nie et al., "Estimating the vegetation canopy height using micro-pulse photon-counting LiDAR data," Opt Express, 26(10): A520-A540 (2018)

[8] U. C. Herzfeld et al., "Surface-Height Determination of Crevassed Glaciers-Mathematical Principles of an Autoadaptive Density-Dimension Algorithm and Validation Using ICESat-2 Simulator (SIMPL) Data", Ieee Transactions on Geoscience and Remote Sensing, 55(4): 1874-1896 (2017). 